11

\title{
Влияние ионосферы на возбуждение электромагнитного поля диапазона КНЧ и более низких частот в ближней зоне
}

\author{
(C) Е.Д. Терещенко, ${ }^{1}$ П.Е. Терещенко, ${ }^{1,2}$ А.Е. Сидоренко, ${ }^{1}$ В.Ф. Григорьев, ${ }^{1}$ А.А. Жамалетдинов ${ }^{2}$ \\ ${ }^{1}$ Полярный геофизический институт, \\ 183010 Мурманск, Россия \\ ${ }^{2}$ Санкт-Петербургский филиал Института земного магнетизма, ионосферы и распространения радиоволн \\ им. Н.В. Пушкова РАН, \\ 199034 Санкт-Петербург, Россия \\ e-mail: tereshchenko@gmail.com
}

(Поступило в Редакцию 3 августа 2017 г.)

Рассмотрен вопрос о возбуждении электромагнитного поля диапазона КНЧ и более низких частот в ближней зоне в волноводе Земля-ионосфера. Экспериментальные исследования показали наличие вариаций амплитуды поля в нижней части КНЧ диапазона и на более низких частотах в различных геофизических условиях при отсутствии вариаций в диапазоне СНЧ. Для оценки факторов, обусловливающих эту особенность поведения поля, предложены теоретические расчеты, показывающие, что при низкой проводимости земли влияние ионосферы в ближней зоне может быть заметным.

DOI: 10.21883/JTF.2018.06.46024.2453

\section{Введение}

Применение наземных контролируемых источников электромагнитных полей КНЧ $(3-30 \mathrm{~Hz})$ и СНЧ $(30-300 \mathrm{~Hz})$ диапазонов имеет перспективы для организации связи на больших расстояниях, глубинного зондирования земли, сейсмического мониторинга и дистанционного контроля состояния ионосферы. Положительные отличительные особенности низкочастотных волн заключаются в крайне малом затухании с расстоянием при распространении в волноводе Земля-ионосфера и в глубоком проникновении в подстилающую среду.

Во многих задачах зондирования природных сред для интерпретации результатов проводить измерения поля необходимо на достаточном удалении от источника. В непосредственной близости от наземного горизонтального источника электромагнитное поле слабо зависит от проводимости земли и ионосферы. Для применения импедансных методов зондирования земли требуется расстояние не менее 5-7 скин-слоев, а для установления характерной зависимости поля от геометрических и электрических параметров волновода Земля-ионосфера необходима дистанция более трех высот эффективного волновода $h$.

Промежуточной области малых расстояний от источника $(0.5-2 h)$ в работах по КНЧ и СНЧ диапазонам уделено крайне мало внимания. Существующие теоретические представления и некоторые численные оценки [1] сводятся в основном к тому, что в этой области зависимость поля от проводимости ионосферы пренебрежимо мала, а обратная высота волновода начинает играть роль множителя в выражениях для амплитуды полей лишь дальше нескольких десятков километров от источника. Зачастую влиянием ионосферы на таких малых расстояниях от источника пренебре- гают [2]. Экспериментальные работы в этой области частот и расстояний немногочисленны $[3,4]$, при этом полученные результаты не полностью согласуются с существующими представлениями на частотах ниже $10 \mathrm{~Hz}$, в частности, наблюдаются вариации амплитуды поля в разные времена года и в различное время суток.

В настоящей работе мы представляем результаты измерений, проведенных в ходе эксперимента FENICS2014, проходившего на Кольском п-ве в 2014 г. [5], в котором в отличие от ранее выполненных экспериментов использовался более мощный передатчик, что позволило исключить влияние как внешних, так и внутренних шумов. В качестве теоретической основы для интерпретации предложено новое решение задачи о возбуждении магнитного поля в плоском волноводе горизонтальной заземленной антенной в строгой электродинамической постановке.

\section{Описание и результаты эксперимента}

Международный комплексный эксперимент FENICS2014 был проведен в августе-сентябре 2014 г. Осуществлялась серия сеансов генерации электромагнитного поля в диапазоне $10^{-2}-200 \mathrm{~Hz}$. В первой половине эксперимента использовалась антенна субширотного направления (L1), а во второй - субмеридионального (L2) (рис. 1). Длина каждой антенны составляет около $100 \mathrm{~km}$. Генерация производилась ежедневно с 01:00 до 05:00 по местному времени на 14 частотах продолжительностью 10-15 min на каждой частоте.

Рассмотрим результаты измерений магнитного поля субширотной антенны, которые были получены в обсерватории ПГИ в п. Ловозеро (рис. 1) 23-29 августа 


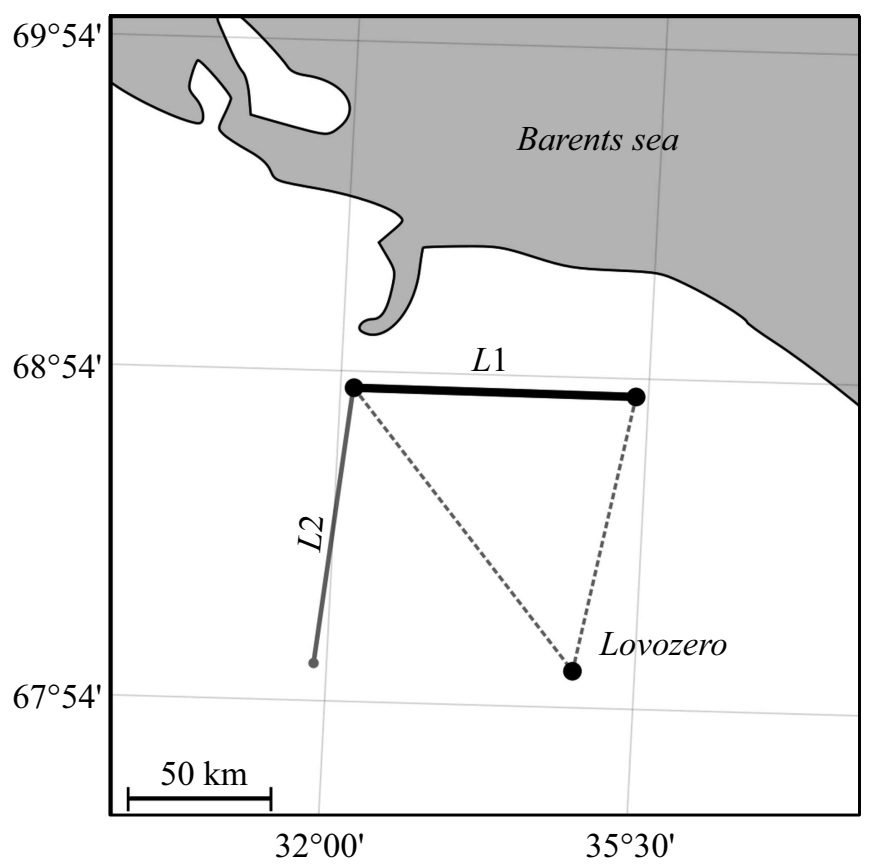

Рис. 1. Карта-схема эксперимента.

2014 г. Расстояния от точки измерений до концов антенны составляли 125 и $100 \mathrm{~km}$.

Регистрация сигналов производилась при помощи трехкомпонентного индукционного магнитометра, имеющего два ортогональных горизонтальных датчика и один вертикальный. Горизонтальные датчики ориентиро-

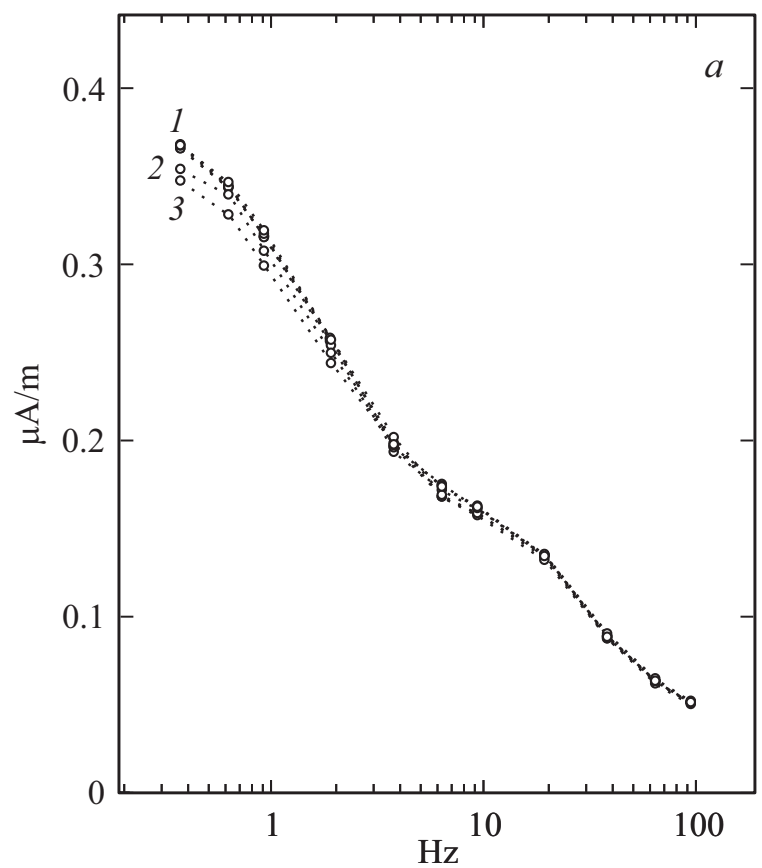

вались по стрелке буссоли в направлениях $\mathrm{C}-\mathrm{Ю}\left(H_{\mathrm{N}-\mathrm{S}}\right)$ и 3-В $\left(H_{\mathrm{W}-\mathrm{E}}\right)$. Магнитное склонение - восточное, $12^{\circ}$. Сила тока в передающей антенне регистрировалась цифровой системой сбора данных. Частота дискретизации составляла $512 \mathrm{~Hz}$. По окончании эксперимента была проведена обработка полученных данных и выполнена нормировка амплитуды магнитного поля на силу тока в антенне.

Результаты измерений в диапазоне $0.4-100 \mathrm{~Hz}$ показаны на рис. 2. Как можно видеть, амплитуда поля в диапазоне частот $10-100 \mathrm{~Hz}$ в течение всего эксперимента оставалась постоянной, а на частотах ниже $10 \mathrm{~Hz}$ средний уровень поля в некоторые дни заметно менялся. Эти колебания амплитуды не могут быть связаны с влиянием случайных шумов - по результатам измерений сигнал превышал шум на два порядка, поэтому измерялся с точностью, многократно превышающей суточные вариации.

Такую же особенность поведения поля мы наблюдали и в предыдущих экспериментах, которые проводились в различные времена года и в разное время суток $[3,4]$.

Для объяснения обнаруженной особенности поведения КНЧ поля необходимо рассмотреть его связь с параметрами нижней ионосферы в области расстояний от источника в пределах одной-двух высот эффективного волновода. С этой целью приведем решение задачи о возбуждении КНЧ-СНЧ поля горизонтальной заземленной антенной с учетом наличия ионосферы, полученное при строгой электродинамической постановке, и выполним сопоставление результатов с экспериментальными наблюдениями.

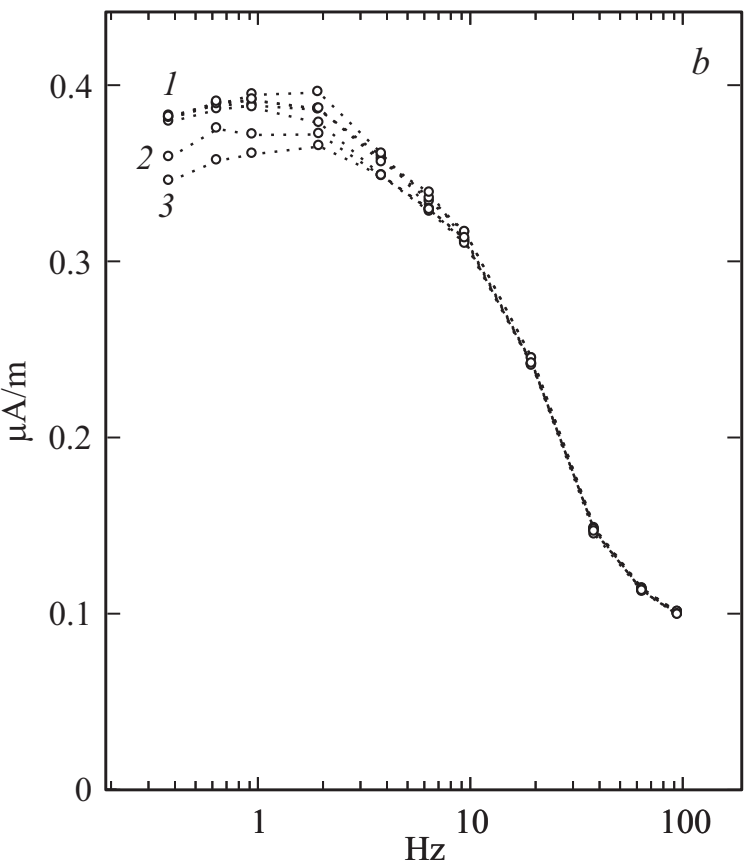

Рис. 2. Зависимость амплитуды магнитного поля от частоты при силе тока в передающей антенне $1 \mathrm{~A}$ в сеансах $23-29$ августа 2014 г. (обс. Ловозеро) - компоненты $H_{\mathrm{W}-\mathrm{E}}(a)$ и $H_{\mathrm{N}-\mathrm{S}}(b)$. Обозначения кривых: 1 - измерения $23-27.08 .2014$ г., 2 - 28.08 .2014 г., $3-29.08 .2014$ г. 


\section{Теоретическая интерпретация экспериментальных результатов}

Рассмотрим возбуждение плоского волновода горизонтальной заземленной антенной. Определим поле в трехслойной среде (рис. 3), формируемое горизонтальным излучателем длиной $2 L$, питаемым током с гармонической зависимостью от времени $\exp (-i \omega t)$ и находящимся на границе раздела $z=0$.

При этом будем считать проводимость земли $\sigma_{-1}$ и ионосферы $\sigma_{1}$ постоянными и изотропными.

Систему координат выберем следующим образом: начало декартовых координат поместим в середину антенны, ось $z$ направим вверх, ось $x$ - вдоль антенны, a $y$ - поперек антенны. Расстояние до точки наблюдения обозначим $R$, а расстояния на плоскости $(x, y, 0)$ обозначим $\rho-$ от центра и $\rho_{\eta}-$ от произвольной точки антенны.

Среду в области $0 \leqslant z \leqslant h$ считаем практически непроводящей $(\sigma=+0$, при этом наличие знака „, + “ нуля указывает на небольшое поглощение) с диэлектрической проницаемостью $\varepsilon_{0} \approx 10^{-9} /(36 \pi) \mathrm{F} / \mathrm{m}$ и магнитной проницаемостью $\mu_{0}=4 \pi \cdot 10^{-7} \mathrm{H} / \mathrm{m}$. Предполагаем, что в области $z<0$ имеем электромагнитные параметры $\varepsilon_{-1}, \mu_{0}, \sigma_{-1}$, а при $z \geqslant h$ - параметры $\varepsilon_{1}, \mu_{0}, \sigma_{1}$.

Задача о возбуждении электромагнитного поля сторонним током $\mathbf{J}$ сводится к решению уравнений Гельмгольца для электрического вектора-потенциала А с соответствующими граничными условиями $[1,2]$. В дальнейшем удобно использовать уравнения для комплексных амплитуд соответствующих компонент $(\mathbf{A} \rightarrow \mathbf{A} \exp (-i \omega t), \mathbf{E} \rightarrow \mathbf{E} \exp (-i \omega t), \mathbf{H} \rightarrow \mathbf{H} \exp (-i \omega t)$, где $\mathbf{E}$ и $\mathbf{H}-$ электрическое и магнитное поля).

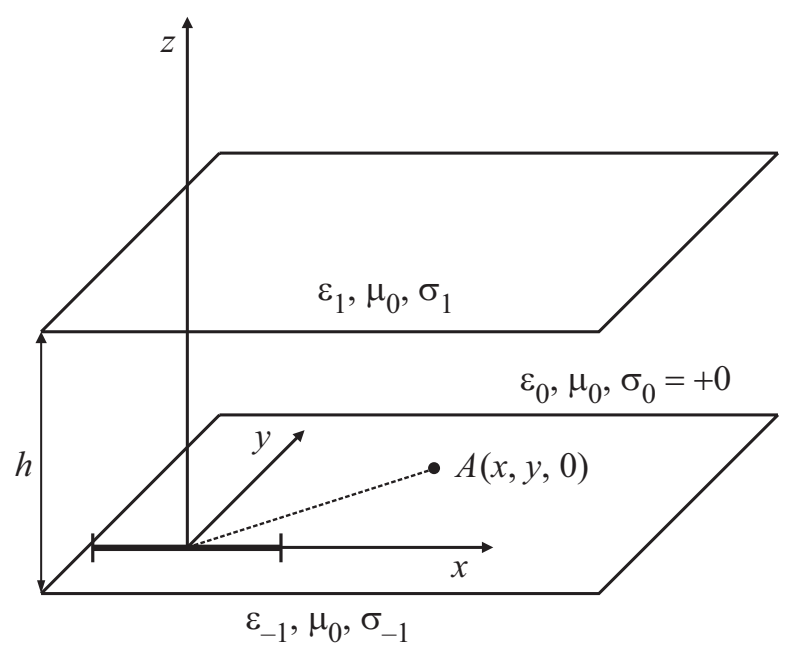

Рис. 3. Геометрическая схема задачи. Земля $-z<0$, воздух $-0 \leqslant z \leqslant h$, ионосфера $-z>h$.
Принимая во внимание, что источник направлен вдоль оси $x$ (рис. 3), представим А в виде двух составляющих

$$
\mathbf{A}^{(j)}=A_{x}^{(j)} \mathbf{e}_{x}+A_{z}^{(j)} \mathbf{e}_{z},
$$

где значок $j=-1,0,1$ указывает на среду, а $\mathbf{e}_{x}$ и $\mathbf{e}_{z}-$ орты, направленные вдоль осей $x$ и $z$ соответственно.

Дальнейший шаг - это получение решения системы уравнений

$$
\nabla^{2} \mathbf{A}^{(j)}+k_{j}^{2} \mathbf{A}^{(j)}=-\mathbf{J}, j=-1,0,1
$$

с граничными условиями

$$
\begin{gathered}
\left.\mathbf{A}^{(1)}\right|_{z=h}=\left.\mathbf{A}^{(0)}\right|_{z=h},\left.\quad \mathbf{A}^{(-1)}\right|_{z=0}=\left.\mathbf{A}^{(0)}\right|_{z=0} \\
\left.\frac{\partial}{\partial z} A_{x}^{(1)}\right|_{z=h}=\left.\frac{\partial}{\partial z} A_{x}^{(0)}\right|_{z=h},\left.\quad \frac{\partial}{\partial z} A_{x}^{(0)}\right|_{z=0}=\left.\frac{\partial}{\partial z} A_{x}^{(-1)}\right|_{z=0} \\
\left.\frac{1}{k_{1}^{2}} \operatorname{div}^{(1)}\right|_{z=h}=\left.\frac{1}{k_{0}^{2}} \operatorname{div}^{(0)}\right|_{z=h} \\
\left.\frac{1}{k_{0}^{2}} \operatorname{div}^{(0)}\right|_{z=0}=\left.\frac{1}{k_{-1}^{2}} \operatorname{div} \mathbf{A}^{(-1)}\right|_{z=0}
\end{gathered}
$$

Кроме того, для исключения волн, приходящих из бесконечности, в силу поглощения в среде, требуем

$$
\left.\mathbf{A}^{(1)}\right|_{R \rightarrow \infty}=0,\left.\quad \mathbf{A}^{(-1)}\right|_{R \rightarrow \infty}=0 .
$$

Волновые числа $k_{j}$, входящие в уравнения (2) и (3), определяются выражением

$$
k_{j}=\frac{\omega}{c} \sqrt{\frac{\varepsilon_{j}}{\varepsilon_{0}}+i \frac{\sigma_{j}}{\omega \varepsilon_{0}}}=\frac{\omega}{c} \sqrt{\tilde{\varepsilon}_{j}^{\prime}}, \quad j=-1,0,1,
$$

где $c$ - скорость света.

В качестве первого шага найдем решение системы (2) с граничными условиями (3) для точечного заземленного горизонтального источника, расположенного в начале координат.

Для этого случая

$$
\begin{gathered}
\mathbf{J}^{(0)}=J \Delta_{x} \delta(x) \delta(y) \delta(z-0) \mathbf{e}_{x}, \\
\mathbf{J}^{(-1)}=J \Delta_{x} \delta(x) \delta(y) \delta(z+0) \mathbf{e}_{y},
\end{gathered}
$$

где $\delta(x)$ - дельта-функция Дирака, $J$ - сила тока, $J \Delta_{x}$ - дипольный момент, $\Delta_{x}$ - длина диполя, стремящаяся к бесконечно малой величине.

Решение системы (2) с граничными условиями (3) удобно строить в цилиндрической системе координат $(\rho, \phi, z)$ в виде разложения по $\cos \frac{m}{2} \phi, m=0,1, \ldots$, образущих полную систему на промежутке $(0,2 \pi)$. Неизвестные функции, зависящие от $\rho$ и $z$ и входящие в разложение, определяются исходя из граничных условий. Опуская промежуточные преобразования и вычисления, можем представить $A_{x}^{(j)}$ и $A_{z}^{(j)}$ в следующем виде:

$$
A_{x}^{(1)}=\frac{J \Delta_{x}}{4 \pi} \int_{0}^{\infty} \alpha_{1} \exp \left(-v_{1} z\right) \mathbf{J}_{0}(\lambda \rho) d \lambda
$$




$$
\begin{gathered}
A_{x}^{(0)}=\frac{J \Delta_{x}}{4 \pi} \int_{0}^{\infty}\left[\left(\frac{\lambda}{v_{0}}+\alpha_{0}\right) \exp \left(-v_{0} z\right)\right. \\
\left.+\beta_{0} \exp \left(v_{0} z\right)\right] \mathrm{J}_{0}(\lambda \rho) d \lambda, \\
A_{x}^{(-1)}=\frac{J \Delta_{x}}{4 \pi} \int_{0}^{\infty}\left(\frac{\lambda}{v_{-1}}+\beta_{-1}\right) \exp \left(v_{-1} z\right) \mathrm{J}_{0}(\lambda \rho) d \lambda .
\end{gathered}
$$

Подобные выражения имеем и для $A_{z}^{(j)}$ :

$$
\begin{gathered}
A_{z}^{(1)}=-\frac{J \Delta_{x}}{4 \pi} \frac{\partial}{\partial x} \int_{0}^{\infty} \eta_{1} \exp \left(-v_{1} z\right) \frac{\mathrm{J}_{0}(\lambda \rho)}{\lambda} d \lambda \\
A_{z}^{(0)}=-\frac{J \Delta_{x}}{4 \pi} \frac{\partial}{\partial x} \int_{0}^{\infty}\left(\eta_{0} \exp \left(-v_{0} z\right)+\gamma_{0} \exp \left(v_{0} z\right)\right) \frac{\mathrm{J}_{0}(\lambda \rho)}{\lambda} d \lambda \\
A_{z}^{(-1)}=-\frac{J \Delta_{x}}{4 \pi} \frac{\partial}{\partial x} \int_{0}^{\infty} \gamma_{-1} \exp \left(\nu_{-1} z\right) \frac{\mathrm{J}_{0}(\lambda \rho)}{\lambda} d \lambda
\end{gathered}
$$

где

$$
\sqrt{k_{j}^{2}-\lambda^{2}}=i \sqrt{\varkappa_{j}^{2}+\lambda^{2}} \equiv i v_{j}, \varkappa_{j}=-i k_{j}
$$

Так как в процессе вычислений фиксировали ветвь корня таким образом, что $\operatorname{Im}\left(\sqrt{k_{j}^{2}-\lambda^{2}}\right)>0$, то $\operatorname{Re}\left(v_{j}\right)>0$.

Система уравнений для неизвестных $\alpha_{j}, \beta_{j}, \eta_{j}$ и $\gamma_{j}$ получается в результате использования уравнений (3). Граничные условия при $z=h$ дают следующую систему уравнений:

$$
\begin{gathered}
\alpha_{1} \exp \left(-v_{1} h\right)-\alpha_{0} \exp \left(-v_{0} h\right)-\beta_{0} \exp \left(v_{0} h\right)= \\
=\frac{\lambda}{v_{0}} \exp \left(-v_{0} h\right) \\
\eta_{1} \exp \left(-v_{1} h\right)-\eta_{0} \exp \left(-v_{0} h\right)-\gamma_{0} \exp \left(v_{0} h\right)=0 \\
-\alpha_{1} v_{1} \exp \left(-v_{1} h\right)+\alpha_{0} v_{0} \exp \left(-v_{0} h\right)-\beta_{0} v_{0} \exp \left(v_{0} h\right)= \\
=-\lambda \exp \left(-v_{0} h\right), \\
\eta_{1} v_{1} k_{0}^{2} \exp \left(-v_{1} h\right)-\eta_{0} v_{0} k_{1}^{2} \exp \left(-v_{0} h\right) \\
+\gamma_{0} v_{0} k_{1}^{2} \exp \left(v_{0} h\right)=\lambda\left[k _ { 1 } ^ { 2 } \left(\left(\frac{\lambda}{v_{0}}+\alpha_{0}\right) \exp \left(-v_{0} h\right)\right.\right. \\
\left.\left.+\beta_{0} \exp \left(v_{0} h\right)\right)-\alpha_{1} k_{0}^{2} \exp \left(-v_{1} h\right)\right]
\end{gathered}
$$

а при $z=0$

$$
\begin{gathered}
\alpha_{0}+\beta_{0}-\beta_{-1}=\lambda \frac{\nu_{0}-v_{-1}}{v_{0} \nu_{-1}}, \\
\alpha_{0}-\beta_{0}+\beta_{-1} \frac{\nu_{-1}}{v_{0}}=0, \\
\eta_{0}+\gamma_{0}-\gamma_{-1}=0,
\end{gathered}
$$

$$
\begin{aligned}
\eta_{0} v_{0} k_{-1}^{2} & +\gamma_{-1} v_{-1} k_{0}^{2}=\lambda\left[k_{0}^{2}\left(\frac{\lambda}{v_{-1}}+\beta_{-1}\right)\right. \\
& \left.-k_{-1}^{2}\left(\frac{\lambda}{v_{0}}+\alpha_{0}\right)\right] .
\end{aligned}
$$

Совместное решение (9) и (10) позволяет определить коэффициенты, входящие в выражение (6). Для того, чтобы иметь представление о структуре коэффициентов, используемых в дальнейшем, приведем выражения для $\alpha_{0}$ и $\beta_{0}$ :

$$
\begin{aligned}
& \alpha_{0}=\frac{\lambda\left(v_{0}-v_{1}\right)}{v_{0}} \\
& \times \frac{\left(v_{0}+v_{1}\right)+\left(v_{0}-v_{1}\right) \exp \left(-2 v_{0} h\right)}{\left(v_{0}+v_{-1}\right)\left(v_{0}+v_{1}\right)-\left(v_{0}-v_{-1}\right)\left(v_{0}-v_{1}\right) \exp \left(-2 v_{0} h\right)}, \\
& \beta_{0}=\frac{2 \lambda\left(v_{0}-v_{1}\right) \exp \left(-2 v_{0} h\right)}{\left(v_{0}+v_{-1}\right)\left(v_{0}+v_{1}\right)-\left(v_{0}-v_{-1}\right)\left(v_{0}-v_{1}\right) \exp \left(-2 v_{0} h\right)} .
\end{aligned}
$$

Имея результаты вычислений для вектора-потенциала, можно определить электромагнитное поле. Чтобы не усложнять расчетов, рассмотрим составляющую магнитного поля $H_{x}$. Используя связь полей с векторомпотенциалом $\mathbf{H}^{(-1)}=\operatorname{rot} \mathbf{A}^{(-1)}$, можно получить

$$
H_{x}^{(-1)}=\frac{\partial}{\partial y} A_{z}^{(-1)} \text {. }
$$

Подставляя выражения для $A_{x}^{(-1)}$ и $A_{z}^{(-1)}$ из (6) и (7), находим

$$
H_{x}^{(-1)}=-\frac{J \Delta_{x}}{4 \pi} \frac{\partial}{\partial x} \frac{\partial}{\partial y} \int_{0}^{\infty} \frac{\gamma_{-1}}{\lambda} \exp \left(\nu_{-1} z\right) \mathrm{J}_{0}(\lambda \rho) d \lambda .
$$

Выразив коэффициенты $\beta_{-1}, \gamma_{-1}$ через $\alpha_{0}, \beta_{0}, \eta_{0}$ и $\gamma_{0}$ с помощью (9) и (10), получим

$$
H_{x}^{(-1)}=-\frac{J \Delta_{x}}{4 \pi} \frac{\partial}{\partial x} \frac{\partial}{\partial y} \int_{0}^{\infty} \frac{\eta_{0}+\gamma_{0}}{\lambda} \exp \left(\nu_{-1} z\right) \mathrm{J}_{0}(\lambda \rho) d \lambda
$$

При возбуждении волн с частотой ниже $300 \mathrm{~Hz}$ xорошим приближением при рассмотрении поля является „Квазистационарное приближение“ $[2,6]$, в рамках которого полагают $\varkappa_{0} \rightarrow 0$. Воспользуемся условием $\varkappa_{0}=0$. Тогда из систем уравнений (9) и (10) следует

$$
\left.\eta_{0}\right|_{\varkappa_{0}=0}=-\left.\left(1+\alpha_{0}\right)\right|_{\varkappa_{0}=0},\left.\gamma_{0}\right|_{\varkappa_{0}=0}=\left.\beta_{0}\right|_{\varkappa_{0}=0} .
$$

Подставляя эти значения в (13), имеем

$$
\begin{aligned}
& \left.H_{x}^{(-1)}\right|_{\varkappa_{0} \rightarrow 0}=\frac{J \Delta_{x}}{4 \pi \varkappa_{-1}} \frac{\partial}{\partial x} \\
& \times \frac{\partial}{\partial y}\left[\int_{0}^{\infty} \frac{2 \varkappa_{-1}}{\lambda+v_{-1}} \exp \left(v_{-1} z\right) \mathrm{J}_{0}(\lambda \rho) d \lambda\right. \\
& \left.+2 \int_{0}^{\infty} \frac{v_{-1}}{\varkappa_{-1}} f\left(\lambda, \varkappa_{-1}, \varkappa_{1}, h\right) \exp \left(v_{-1} z\right) \mathrm{J}_{0}(\lambda \rho) d \lambda\right],
\end{aligned}
$$


где

$$
\begin{aligned}
& f\left(\lambda, \varkappa_{-1}, \varkappa_{1}, h\right)= \\
& =\frac{2\left(\lambda-v_{-1}\right)\left(\lambda-v_{1}\right) \exp (-2 \lambda h)}{\left(\lambda+v_{-1}\right)\left(\lambda+v_{1}\right)-\left(\lambda-v_{-1}\right)\left(\lambda-v_{1}\right) \exp (-2 \lambda h)} .
\end{aligned}
$$

Формула (15) представляет поле в виде суммы поля в двуслойной среде и дополнения, отражающего влияние ионосферы. При $h \rightarrow \infty$, т. е. при отсутствии ионосферы, второе слагаемое в скобках стремится к нулю.

Первое слагаемое в скобках несложно вычислить, используя два интеграла Ватсона [7]:

$$
\begin{gathered}
\int_{0}^{\infty} \frac{\exp \left(v_{-1} z\right)}{v_{-1}} \mathrm{~J}_{0}(\lambda \rho)=\frac{\exp \left(-\varkappa_{-1} R\right)}{R}=\frac{\exp \left(i k_{-1} R\right)}{R}, \\
\int_{0}^{\infty} \frac{\exp \left(v_{-1} z\right)}{v_{-1}} \mathrm{~J}_{0}(\lambda \rho)=\mathrm{I}_{0}\left(r_{+}\right) \mathrm{K}_{0}\left(r_{-}\right)
\end{gathered}
$$

где

$$
R=\sqrt{\rho^{2}+z^{2}}, \quad r_{+}=\varkappa_{-1} \frac{R+z}{2}, \quad r_{-}=\varkappa_{-1} \frac{R-z}{2},
$$

a $\mathrm{I}_{0}\left(r_{+}\right)$и $\mathrm{K}_{0}\left(r_{-}\right)$- модифицированные функции Бесселя.

В результате получим

$$
\begin{aligned}
& \left.H_{x}^{(-1)}\right|_{\varkappa_{0} \rightarrow 0}=-\frac{J \Delta_{x}}{4 \pi \varkappa_{-1}} \frac{\partial}{\partial x} \frac{\partial}{\partial y}\left[\frac{1}{\varkappa_{-1}} \frac{\partial}{\partial z}\right. \\
& \times \frac{\exp \left(\varkappa_{-1} R\right)}{R}-\frac{1}{\varkappa_{-1}} \frac{\partial^{2}}{\partial z^{2}} \mathrm{I}_{0}\left(r_{+}\right) \mathrm{K}_{0}\left(r_{-}\right) \\
& \left.-2 \int_{0}^{\infty} \frac{\nu_{-1}}{\varkappa_{-1}} f\left(\lambda, \varkappa_{-1}, \varkappa_{1}, h\right) \exp \left(\nu_{-1} z\right) \mathrm{J}_{0}(\lambda \rho) d \lambda\right] .
\end{aligned}
$$

Отсюда следует выражение для поля на границе раздела $z=0$

$$
\begin{aligned}
& \left.H_{x}^{(-1)}\right|_{\varkappa_{0} \rightarrow 0, z \rightarrow 0}=\frac{J \Delta_{x}}{4 \pi \varkappa_{-1}} \frac{\partial}{\partial x} \frac{\partial}{\partial y}\left(\varkappa _ { - 1 } \left[\mathrm{I}_{0}\left(\rho \frac{\varkappa_{-1}}{2}\right)\right.\right. \\
& \left.\times \mathrm{K}_{0}\left(\rho \frac{\varkappa_{-1}}{2}\right)+\mathrm{I}_{1}\left(\rho \frac{\varkappa_{-1}}{2}\right) \mathrm{K}_{1}\left(\rho \frac{\varkappa_{-1}}{2}\right)\right] \\
& \left.+2 \int_{0}^{\infty} \frac{\nu_{-1}}{\varkappa_{-1}} f\left(\lambda, \varkappa_{-1}, \varkappa_{1}, h\right) \exp \left(v_{-1} z\right) \mathrm{J}_{0}(\lambda \rho) d \lambda\right)\left.\right|_{z \rightarrow 0} .
\end{aligned}
$$

Принимая во внимание, что

находим

$$
\frac{\partial}{\partial y}=\frac{\partial \rho}{\partial y} \frac{\partial}{\partial \rho}=\frac{y}{\rho} \frac{\partial}{\partial \rho}
$$

$$
\begin{aligned}
& \left.H_{x}^{(-1)}\right|_{\varkappa_{0} \rightarrow 0, z \rightarrow 0}=-\frac{J \Delta_{x}}{2 \pi \varkappa_{-1}} \frac{\partial}{\partial x} \frac{y}{\rho}\left[\frac{\varkappa_{-1}}{\rho} \mathrm{I}_{1}\left(\rho \frac{\varkappa_{-1}}{2}\right)\right. \\
& \left.\times \mathrm{K}_{1}\left(\rho \frac{\varkappa_{-1}}{2}\right)+\int_{0}^{\infty} \frac{\nu_{-1}}{\varkappa_{-1}} f\left(\lambda, \varkappa_{-1}, \varkappa_{1}, h\right) \mathrm{J}_{1}(\lambda \rho) \lambda d \lambda\right] .
\end{aligned}
$$

Введем новую переменную интегрирования $s=\lambda \rho$ и обозначим

$$
\rho \varkappa_{j}=(1-i) \rho \sqrt{\pi f \mu_{0} \sigma_{j}} \equiv(1-i) D_{j}, \quad j= \pm 1 .
$$

Нетрудно заметить, что $D_{j}-$ это отношение расстояния до точки наблюдения к толщине скин-слоя в среде. Таким образом, в новых переменных будем иметь следующие выражения для поля:

$$
\left.H_{x}^{(-1)}\right|_{\varkappa_{0} \rightarrow 0, z \rightarrow 0}=-\frac{J \Delta_{x}}{2 \pi} \frac{\partial}{\partial x} \frac{y}{\rho^{2}} \frac{F_{H}(\rho)}{(1-i) D_{-1}},
$$

где

$$
\begin{array}{r}
F_{H}(\rho)=(1-i) D_{-1} \mathrm{I}_{1}\left(D_{-1} \frac{1-i}{2}\right) \mathrm{K}_{1}\left(D_{-1} \frac{1-i}{2}\right) \\
+\int_{0}^{\infty} \sqrt{1-\frac{s^{2}}{2 i D_{-1}^{2}}} \tilde{f}(s) \mathrm{J}_{1}(s) d s,
\end{array}
$$

$$
\begin{aligned}
& \tilde{f}(s)= \\
& =\frac{2 s\left(s-\sqrt{s^{2}-2 i D_{-1}^{2}}\right)\left(s-\sqrt{s^{2}-2 i D_{1}^{2}}\right) \exp (-2 s h / \rho)}{\left(s+\sqrt{s^{2}-2 i D_{-1}^{2}}\right)\left(s+\sqrt{s^{2}-2 i D_{1}^{2}}\right)-} . \\
& \quad-\left(s-\sqrt{s^{2}-2 i D_{-1}^{2}}\right)\left(s-\sqrt{s^{2}-2 i D_{1}^{2}}\right) \exp (-2 s h / \rho)
\end{aligned}
$$

Формула (18) описывает поле горизонтального заземленного диполя. Поле линейной антенны определяется суммой полей, излучаемых источниками, относящимися к антенне. Обозначим $\mathbf{H}^{(-1)}(\rho, z)$ магнитное поле, возбуждаемое линейной антенной в нижнем полупространстве, тогда

$$
\mathbf{H}^{(-1)}(\rho, z=0)=\sum \mathbf{H}^{(-1)}(\rho, z \rightarrow 0),
$$

т. е. равно сумме полей источников, находящихся в точке $\eta$ на антенне (рис. 3). Устремляя $\Delta_{x}$ к нулю, суммирование в формуле можно заменить на интегрирование по $\eta$, тогда $J \Delta_{x} \rightarrow J d \eta$. В результате получим

$$
\mathscr{H}_{x}^{(-1)}(\rho, 0)=\left.\int_{-L}^{L} H_{x}^{(-1)}\left(\rho_{\eta}, z \rightarrow 0\right)\right|_{J \Delta_{x} \rightarrow I} d \eta,
$$

где $\rho_{\eta}=\sqrt{(x-\eta)^{2}+y^{2}}$.

Для интегрирования необходимо подставить выражение для поля диполя, находящегося не в начале координат, а в точке $x=\eta$. С этой целью произведем замену в выражении (18) для магнитного поля $x \rightarrow x-\eta, \rho \rightarrow \rho_{\eta}$ и $\partial / \partial x=-\partial / \partial \eta$.

Тогда, выполнив интегрирование по $\eta$, получим

$$
\mathscr{H}_{x}^{(-1)}(\rho, 0)=\left.\frac{I}{2 \pi} \frac{y}{\rho^{2}} \frac{F_{H}\left(\rho_{\eta}\right)}{(1-i) D_{-1}}\right|_{-L} ^{L} .
$$



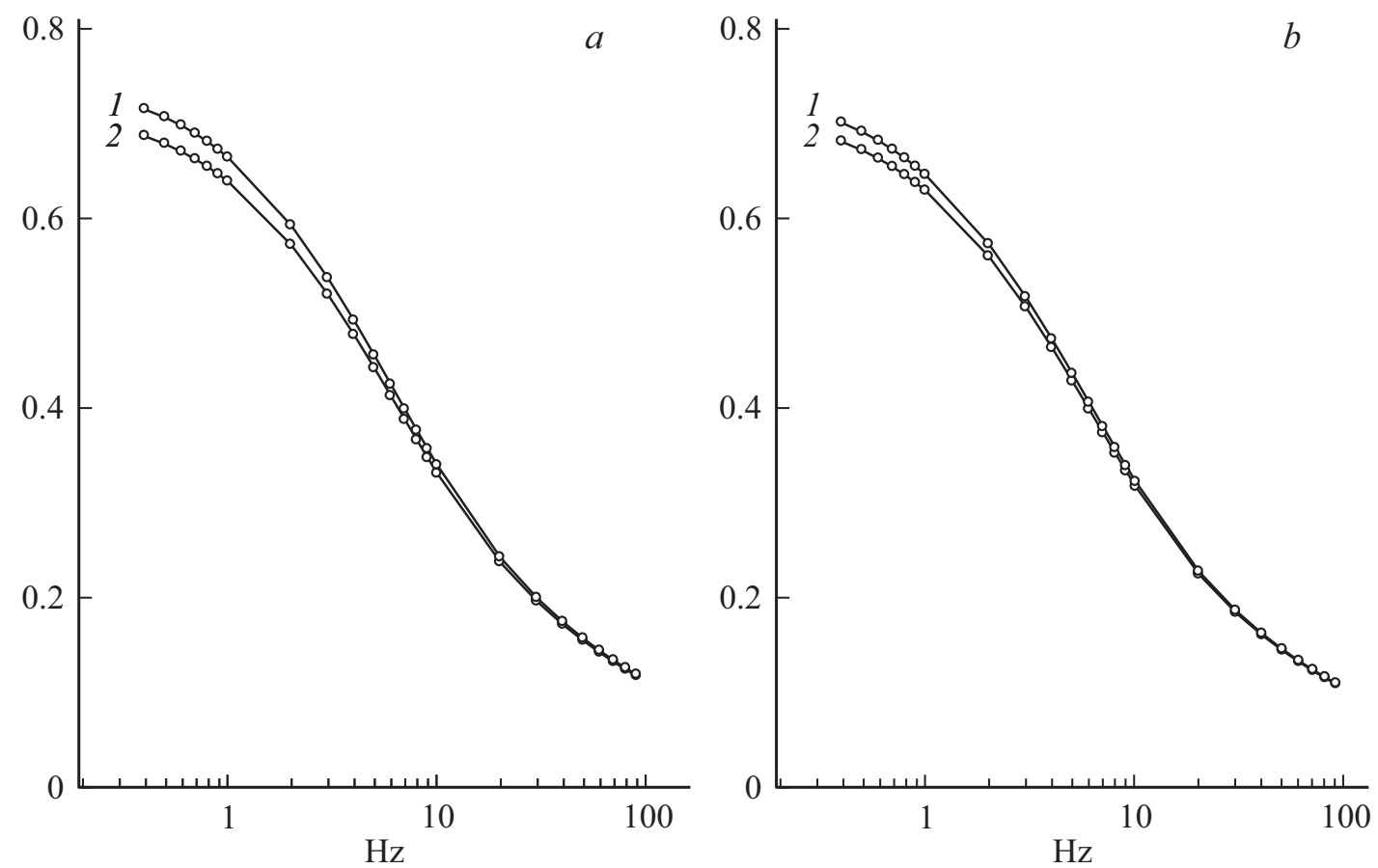

Рис. 4. Расчетные значения $F_{h} / D_{-1}$ при высотах ионосферы $h=70 \mathrm{~km}(a)$ и $85 \mathrm{~km}(b)$ и проводимостях $\sigma_{1}=10^{-4} \mathrm{Sm} / \mathrm{m}$ (кривые 1) и $5 \cdot 10^{-4} \mathrm{Sm} / \mathrm{m}$ (кривые 2$)$.

Таким образом, связь амплитуды поля с условиями внешней среды определяется множителем $F_{H} / D_{-1}$, зависящим от $\sigma_{-1}, \sigma_{1}$, и $h$. На рис. 4 приведены графики этой величины для частот $0.4-100 \mathrm{~Hz}$ для значений параметров $\sigma_{1}=10^{-4}, 5 \cdot 10^{-4} \mathrm{Sm} / \mathrm{m}$ и $h=70$ и $85 \mathrm{~km}$. Координаты точки, для которой выполнен расчет, соответствуют условиям рассматриваемого экперимента, а проводимость земли $\sigma_{-1}$ принималась равной $10^{-5} \mathrm{Sm} / \mathrm{m}$, что характерно для Кольского п-ва.

Как следует из графиков, изменения высоты ионосферы влияют на общий уровень амплитуды во всем диапазоне, в то время как вариации эффективной проводимости изменяют амплитуду лишь в некоторой области частот, не превышающей $10 \mathrm{~Hz}$. Такой же характер имеют и вариации амплитуды в различные дни наблюдений во время эксперимента (рис. 2).

\section{Выводы}

Предложенное в работе решение задачи о поле горизонтального диполя в трехслойной среде позволяет связать наблюдаемые в экспериментах в КНЧ диапазоне вариации амплитуды магнитного поля контролируемого источника на расстояниях 1-2 высот эффективного волновода с изменениями проводимости ионосферы в различных геофизических условиях. По данным ближайшей обсерватории Лопарская (Мурманская обл.) $K$-индекс геомагнитной активности в часы наблюдений 23-27 августа колебался в пределах от нуля до единицы, а 28-29 августа вырос до пяти-шести единиц. Как видно на рис. 4, кривые для спокойных дней практически совпадали друг с другом, а в возмущенный период их уровень понижался. На основании этого можно предположить, что возмущение привело к изменению концентрации электронов и проводимости в ионосфере.

Таким образом, экспериментально обнаружено и теоретически показано, что в области, не превышающей 1-2 высот волновода, имеется заметное влияние ионосферы на амплитуду электромагнитного поля диапазона КНЧ и более низких частот. Предложенный в работе расчет поля справедлив и на больших расстояниях - до $2000 \mathrm{~km}$, где допустимо пренебрежение кривизной волновода Земля-ионосфера. В результате расширяются возможности интерпретации дистанционного зондирования как ионосферы, так и земли с использованием контролируемых наземных источников КНЧ диапазона, а также повышается их информативность.

\section{Список литературы}

[1] Wait J.R. Electromagnetic Waves in Stratified Media. Elmsford, N.Y:: Pergamon Press, 1970.

[2] Вешев А.В. Электропрофилирование на постоянном и переменном токе. 2-е изд. перераб. и доп. Л.: Недра, 1980. $391 \mathrm{c.}$

[3] Терещенко Е.Д., Григорьев В.Ф., Сидоренко А.Е., Миличенко А.Н., Мольков А.В., Собчаков Л.А., Васильев А.В. // Письма в ЖЭТФ. 2007. Т. 85. Вып. 8. С. 471-473. 
[4] Терещенко Е.Д., Григорьев В.Ф., Сидоренко А.Е., Миличенко А.Н., Мольков А.В., Собчаков Л.А., Васильев А.В. // Геомагнетизм и аэрономия. 2007. Т. 47. № 6. С. 855-856.

[5] Колобов В.В., Баранник М.Б., Жамалетдинов А.А. // Труды КНЦ. 2/2015 (28). Вып. 10. С. 52-64.

[6] Fock $V$. Zur Berechnung des elektromagnetischen Wechselstromfeldes bei ebener Begrenzung. Ann. Phys. 1933. Vol. 409. P. 401-420. doi: 10.1002/andp.19334090405

[7] Терещенко Е.Д., Терещенко П.Е. // ЖТФ. 2017. Т. 87. Вып. 3. C. 453-457. doi: 10.21883/JTF.2017.03.44254.1917 